\title{
Story Cloze Task: UW NLP System
}

\author{
Roy Schwartz ${ }^{1,2}$, Maarten Sap ${ }^{1}$, Ioannis Konstas ${ }^{1}$, \\ Leila Zilles ${ }^{1}$, Yejin Choi $^{1}$ and Noah A. Smith ${ }^{1}$
}

\author{
${ }^{1}$ Computer Science \& Engineering, University of Washington, Seattle, WA 98195, USA \\ ${ }^{2}$ Allen Institute for Artificial Intelligence, Seattle, WA 98103, USA \\ \{roysch, msap, ikonstas, lzilles, yejin, nasmith\}@es.washington.edu
}

\begin{abstract}
This paper describes University of Washington NLP's submission for the Linking Models of Lexical, Sentential and Discourse-level Semantics (LSDSem 2017) shared task-the Story Cloze Task. Our system is a linear classifier with a variety of features, including both the scores of a neural language model and style features. We report $75.2 \%$ accuracy on the task. A further discussion of our results can be found in Schwartz et al. (2017).
\end{abstract}

\section{Introduction}

As an effort to advance commonsense understanding, Mostafazadeh et al. (2016) developed the story cloze task, which is the focus of the LSDSem 2017 shared task. In this task, systems are given two short, self-contained stories, which differ only in their last sentence: one has a right (coherent) ending, and the other has a wrong (incoherent) ending. The task is to tell which is the right story. In addition to the task, the authors also introduced the ROC story corpus - a training corpus of five-sentence (coherent) stories. Table 1 shows an example of a coherent story and an incoherent story from the story cloze task.

In this paper, we describe University of Washington NLP's submission for the shared task. Our system explores several types of features for the task. First, we train a neural language model (Mikolov et al., 2010) on the ROC story corpus. We use the probabilities assigned by the model to each of the endings (right and wrong) as classification features.

Second, we attempt to distinguish between right and wrong endings using style features, such as sentence length, character $n$-grams and word $n$ -

\begin{tabular}{|l|l|}
\hline Story Prefix & Ending \\
\hline $\begin{array}{l}\text { Kathy went shopping. } \\
\text { She found a pair of } \\
\text { great shoes. The } \\
\text { shoes were \$300. She } \\
\text { bought the shoes. }\end{array}$ & $\begin{array}{l}\text { She felt buyer's re- } \\
\text { morse after the pur- } \\
\text { chase. }\end{array}$ \\
\cline { 2 - 2 } & $\begin{array}{l}\text { Kathy hated buying } \\
\text { shoes. }\end{array}$ \\
\hline
\end{tabular}

Table 1: Examples of stories from the story cloze task (Mostafazadeh et al., 2016). The left column shows that first four sentences of a story. The right column shows two contrastive endings for the story: a coherent ending (upper row) and a incoherent one (bottom row).

grams. Our intuition is that the right endings use a different style compared to the wrong endings. The features we use were shown useful for style detection in tasks such as age (Schler et al., 2006), gender (Argamon et al., 2003), and authorship profiling (Stamatatos, 2009).

We feed our features to a logistic regression classifier, and evaluate our system on the shared task. Our system obtains $75.2 \%$ accuracy on the test set. Our findings hint that the different writing tasks used to create the story cloze task-writing right and wrong endings-impose different writing styles on authors. This is further discussed in Schwartz et al. (2017).

\section{System Description}

We design a system that predicts, given a pair of story endings, which is the right one and which is the wrong one. Our system applies a linear classifier guided by several types of features to solve the task. We describe the system in detail below. 


\subsection{Model}

We train a binary logistic regression classifier to distinguish between right and wrong stories. We use the set of right stories as positive samples and the set of wrong stories as negative samples. At test time, for a given pair, we consider the classification results of both candidates. If our classifier assigns different labels to each candidate, we keep them. If not, the label whose posterior probability is lower is reversed. We describe the classification features below.

\subsection{Features}

We use two types of features, designed to capture different aspects of the problem. We use neural language model features to leverage corpus level word distributions, specifically longer term sequence probabilities. We use stylistic features to capture differences in writing between coherent story endings and incoherent ones.

Language model features. We experiment with state-of-the-art text comprehension models, specifically an LSTM (Hochreiter and Schmidhuber, 1997) recurrent neural network language model (RNNLM; Mikolov et al., 2010). Our RNNLM is used to generate two different probabilities: $p_{\theta}$ (ending), which is the language model probability of the fifth sentence alone and $p_{\theta}$ (ending | story), which is the RNNLM probability of the fifth sentence given the first four sentences. We use both of these probabilities as classification features.

In addition, we also apply a third feature:

$$
\frac{p_{\theta}(\text { ending } \mid \text { story })}{p_{\theta}(\text { ending })}
$$

The intuition is that a correct ending should be unsurprising (to the model) given the four preceding sentences of the story (the numerator), controlling for the inherent surprise of the words in that ending (the denominator). ${ }^{1}$

Stylistic features. We hypothesize that right and wrong endings might be distinguishable using style features. We adopt style features that have been shown useful in the past in tasks such as detection of age (Schler et al., 2006; Rosenthal and McKeown, 2011; Nguyen et al., 2011), gender

\footnotetext{
${ }^{1}$ Note that taking the logarithm of the expression in Equation 1 gives the pointwise mutual information between the story and the ending, under the language model.
}

(Argamon et al., 2003; Schler et al., 2006; Bamman et al., 2014), and native language (Koppel et al., 2005; Tsur and Rappoport, 2007; Bergsma et al., 2012).

We add the following classification features to capture style differences between the two endings. These features are computed on the story endings alone (right or wrong), and do not consider, either at train or at test time, the first four (shared) sentences of each story.

- Length. The number of words in the sentence.

- Word n-grams. We use sequences of 15 words. Following Tsur et al. (2010) and Schwartz et al. (2013), we distinguish between high frequency and low frequency words. Specifically, we replace content words, which are often low frequency, with their part-of-speech tags (Nouns, Verbs, Adjectives, and Adverbs).

- Character n-grams. Character $n$-grams are useful features in the detection of author style (Stamatatos, 2009) or language identification (Lui and Baldwin, 2011). We use character 4-grams.

\subsection{Experimental Setup}

The story cloze task doesn't have a training corpus for the right and wrong endings. Therefore, we use the development set as our training set, holding out $10 \%$ for development (3,366 training endings, 374 for development). We keep the story cloze test set as is (3,742 endings).

We use Python's sklearn logistic regression implementation with $L_{2}$ regularization, performing grid search on the development set to tune a single hyperparameter - the regularization parameter.

For computing the RNN features, we start by tokenizing the text using the nltk tokenizer. ${ }^{2}$ We then use TensorFlow ${ }^{3}$ to train the RNNLM using a single-layer LSTM of hidden dimension 512. We use the ROC Stories for training, setting aside 10\% for validation of the language model. ${ }^{4}$ We replace all words occurring less than 3 times by a special out-of-vocabulary character, yielding a vocabulary size of 21,582. Only during training, we apply a

\footnotetext{
${ }^{2}$ www.nltk.org/api/nltk.tokenize.html

${ }^{3}$ www.tensorflow. org

${ }^{4}$ We train on both the Spring 2016 and the Winter 2017 datasets, a total of roughly $100 \mathrm{~K}$ stories.
} 


\begin{tabular}{|l|r|}
\hline Model & Acc. \\
\hline DSSM (Mostafazadeh et al., 2016) & 0.585 \\
LexVec (Salle et al., 2016) & 0.599 \\
\hline \hline RNNLM features & 0.677 \\
Stylistic features & 0.724 \\
Combined (Style + RNNLM) & $\mathbf{0 . 7 5 2}$ \\
\hline \hline Human judgment & 1.000 \\
\hline
\end{tabular}

Table 2: Results on the test set of the story cloze task. The first block are published results, the second block are our results. LexVec results are taken from (Speer et al., 2017). Human judgement scores are taken from (Mostafazadeh et al., 2016).

dropout rate of $60 \%$ while running the LSTM over all 5 sentences of the stories. Using Adam optimizer (Kingma and $\mathrm{Ba}, 2015$ ) and a learning rate of $\eta=.001$, we train to minimize cross-entropy. The resulting RNN features (see Section 2.2) are taken in log space.

For the style features, we add a START symbol at the beginning of each sentence. ${ }^{5}$ We keep $n$-gram (character or word) features that occur at least five times in the training set. All stylistic feature values are normalized to the range $[0,1]$. For the part-ofspeech features, we tag all endings with the Spacy POS tagger. ${ }^{6}$ The total number of features used by our system is 7,651 .

\section{Results}

The performance of our system is described in Table 2 . With $75.2 \%$ accuracy, our system achieves $15.3 \%$ better than the published state of the art (Salle et al., 2016). The table also shows an analysis of the different features types used by our system. While our RNNLM features alone reach $67.7 \%$, the style features perform better-72.4\% This suggests that while this task is about story understanding, there is some information contained in stylistic features, which are slightly less sensitive to content. As expected, the RNNLM features complement the stylistic ones, boosting performance by $7.5 \%$ (over the RNNLM features) and $2.8 \%$ (over the style features).

In an attempt to provide explanation to the strong performance of the stylistic feature, we hypothesize that the different writing tasks-writing a right and a wrong ending-impose a different

\footnotetext{
${ }^{5}$ Virtually all sentences end with a period or an exclamation mark, so we do not add a STOP symbol.

${ }^{6}$ spacy.io/
}

style on the authors, which is expressed in the different style adopted in each of the cases. The reader is referred to Schwartz et al. (2017) for more details and discussion.

\section{Conclusion}

This paper described University of Washington NLP's submission to the LSDSem 2017 Shared Task. Our system leveraged both neural language model features and stylistic features, achieving $75.2 \%$ accuracy on the classification task.

\section{Acknowledgments}

The authors thank the shared task organizers and anonymous reviewers for feedback. This research was supported in part by DARPA under the Communicating with Computers program.

\section{References}

Shlomo Argamon, Moshe Koppel, Jonathan Fine, and Anat Rachel Shimoni. 2003. Gender, genre, and writing style in formal written texts. Text, 23(3):321-346.

David Bamman, Jacob Eisenstein, and Tyler Schnoebelen. 2014. Gender identity and lexical variation in social media. Journal of Sociolinguistics, 18(2):135-160.

Shane Bergsma, Matt Post, and David Yarowsky. 2012. Stylometric analysis of scientific articles. In Proceedings of the 2012 Conference of the North American Chapter of the Association for Computational Linguistics: Human Language Technologies, pages 327-337, Montréal, Canada, June. Association for Computational Linguistics.

Sepp Hochreiter and Jürgen Schmidhuber. 1997. Long short-term memory. Neural Computation, 9(8):1735-1780.

Diederik Kingma and Jimmy Ba. 2015. Adam: A method for stochastic optimization. In Proceedings of the International Conference on Learning Representations, San Diego, California, USA.

Moshe Koppel, Jonathan Schler, and Kfir Zigdon. 2005. Determining an author's native language by mining a text for errors. In Proceedings of the Eleventh ACM SIGKDD International Conference on Knowledge Discovery in Data Mining, pages 624-628, Chicago, Illinois, USA. Association for Computing Machinery.

Marco Lui and Timothy Baldwin. 2011. Cross-domain feature selection for language identification. In Proceedings of 5th International Joint Conference on 
Natural Language Processing, pages 553-561, Chiang Mai, Thailand, November. Asian Federation of Natural Language Processing.

Tomáš Mikolov, Martin Karafiát, Lukáš Burget, Jan Černocký, and Sanjeev Khudanpur. 2010. Recurrent neural network based language model. In Proceedings of the 11th Annual Conference of the International Speech Communication Association, pages 1045-1048, Makuhari, Chiba, Japan. International Speech Communication Association.

Nasrin Mostafazadeh, Nathanael Chambers, Xiaodong He, Devi Parikh, Dhruv Batra, Lucy Vanderwende, Pushmeet Kohli, and James Allen. 2016. A corpus and cloze evaluation for deeper understanding of commonsense stories. In Proceedings of the 2016 Conference of the North American Chapter of the Association for Computational Linguistics: Human Language Technologies, pages 839-849, San Diego, California, USA, June. Association for Computational Linguistics.

Dong Nguyen, Noah A. Smith, and Carolyn P. Rosé. 2011. Author age prediction from text using linear regression. In Proceedings of the 5th ACLHLT Workshop on Language Technology for Cultural Heritage, Social Sciences, and Humanities, pages 115-123, Portland, Oregon, USA, June. Association for Computational Linguistics.

Sara Rosenthal and Kathleen McKeown. 2011. Age prediction in blogs: A study of style, content, and online behavior in pre- and post-social media generations. In Proceedings of the 49th Annual Meeting of the Association for Computational Linguistics: Human Language Technologies, pages 763772, Portland, Oregon, USA, June. Association for Computational Linguistics.

Alexandre Salle, Marco Idiart, and Aline Villavicencio. 2016. Enhancing the lexvec distributed word representation model using positional contexts and external memory. arXiv:1606.01283.

Jonathan Schler, Moshe Koppel, Shlomo Argamon, and James Pennebaker. 2006. Effects of age and gender on blogging. In AAAI Spring Symposium: Computational Approaches to Analyzing Weblogs, pages 199-205, Palo Alto, California, USA, March. AAAI Press.

Roy Schwartz, Oren Tsur, Ari Rappoport, and Moshe Koppel. 2013. Authorship attribution of micromessages. In Proceedings of the 2013 Conference on Empirical Methods in Natural Language Processing, pages 1880-1891, Seattle, Washington, USA, October. Association for Computational Linguistics.

Roy Schwartz, Maarten Sap, Ioannis Konstas, Leila Zilles, Yejin Choi, and Noah A. Smith. 2017. The effect of different writing tasks on linguistic style: A case study of the ROC story cloze task. arXiv:1702.01841.
Robert Speer, Joshua Chin, and Catherine Havasi. 2017. Conceptnet 5.5: An open multilingual graph of general knowledge. In Proceedings of the 31 st AAAI Conference on Artificial Intelligence, San Francisco, California, USA, February. AAAI Press.

Efstathios Stamatatos. 2009. A survey of modern authorship attribution methods. Journal of the American Society for information Science and Technology, 60(3):538-556.

Oren Tsur and Ari Rappoport. 2007. Using classifier features for studying the effect of native language on the choice of written second language words. In Proceedings of the Workshop on Cognitive Aspects of Computational Language Acquisition, pages 916, Prague, Czech Republic, June. Association for Computational Linguistics.

Oren Tsur, Dmitry Davidov, and Ari Rappoport. 2010. ICWSM - a great catchy name: Semi-supervised recognition of sarcastic sentences in online product reviews. In Proceedings of the Fourth International AAAI Conference on Weblogs and Social Media, pages 162-169, Washington, DC, USA, May. AAAI Press. 